\title{
An endogenous artificial microRNA system for unraveling the function of root endosymbioses related genes in Medicago truncatula
}

\author{
Emanuel A Devers ${ }^{1,2+}$, Julia Teply ${ }^{1 \dagger}$, Armin Reinert ${ }^{1}$, Nicole Gaude ${ }^{1}$ and Franziska Krajinski ${ }^{*}$
}

\begin{abstract}
Background: Legumes have the unique capacity to undergo two important root endosymbioses: the root nodule symbiosis and the arbuscular mycorrhizal symbiosis. Medicago truncatula is widely used to unravel the functions of genes during these root symbioses. Here we describe the development of an artificial microRNA (amiR)-mediated gene silencing system for $M$. truncatula roots.

Results: The endogenous microRNA (miR) mtr-miR159b was selected as a backbone molecule for driving amiR expression. Heterologous expression of mtr-miR159b-amiR constructs in tobacco showed that the backbone is functional and mediates an efficient gene silencing. amiR-mediated silencing of a visible marker was also effective after root transformation of $M$. truncatula constitutively expressing the visible marker. Most importantly, we applied the novel amiR system to shed light on the function of a putative transcription factor, MtErf1, which was strongly induced in arbuscule-containing cells during mycorrhizal symbiosis. MtPt4 promoter driven amiR-silencing led to strongly decreased transcript levels and deformed, non-fully truncated arbuscules indicating that MtErf1 is required for arbuscule development.
\end{abstract}

Conclusions: The endogenous amiR system demonstrated here presents a novel and highly efficient tool to unravel gene functions during root endosymbioses.

\section{Background}

In the past decades, legumes have been established as important model systems to discover the molecular and physiological background of the root nodule and arbuscular mycorrhizal symbiosis. Analysis of gene function during root endosymbioses requires reverse genetics approaches based on expression perturbation experiments. In the past, RNA interference (RNAi) or virus induced gene silencing (VIGS) has been widely applied to produce plant knock-down mutants. Both systems exploit endogenous posttranscriptional gene silencing (PTGS) pathways of eukaryotes [1-7].

An efficient VIGS system has not yet been established for M. truncatula, hence RNAi approaches have been widely applied to elucidate gene functions in Agrobacterium

\footnotetext{
* Correspondence: krajinski@mpimp-golm.mpg.de

${ }^{\dagger}$ Equal contributors

${ }^{1}$ Max Planck Institute of Molecular Plant Physiology, Am Muehlenberg 1

14476, Potsdam (OT) Golm, Germany

Full list of author information is available at the end of the article
}

rhizogenes transformed roots. However, previous knockdown approaches in this system using RNAi constructs often did not lead to consistent results due off-target effects of RNAi approaches. RNAi is based on a hairpin construct with short inverted sequence fragments of the gene of interest separated by an intron and is processed via the IRPTGS pathway. The expressed RNA folds into a perfect matched double strand and is processed by DCL4 to short interfering RNAs (siRNAs). However, in some cases the approach is limited by inefficient knock down of the target gene in legumes due to unknown causes [8]. Additionally, the RNAi approach leads to heterogeneous accumulation of siRNA products, derived from the expressed hairpin which can lead to unspecific downregulation of related genes (off-targets), especially in large gene families with high sequence similarity [9]. Also, a mechanism called transitivity leads to an amplification and spreading of the siRNA species, yielding secondary siRNAs independent of the primary siRNA signal [10]. These
C Biomed Central

(C) 2013 Devers et al.; licensee BioMed Central Ltd. This is an Open Access article distributed under the terms of the Creative Commons Attribution License (http://creativecommons.org/licenses/by/2.0), which permits unrestricted use, distribution, and reproduction in any medium, provided the original work is properly cited. 
secondary siRNAs cover sequence information outside of the designed RNAi construct, thus enhancing off-target effects. There is precedent for artificial miRNAs to be more specific as RNAi constructs $[11,12]$, here we suggest artificial miRNAs as an alternative tool for gene knock down approaches. However, we do not provide a direct comparison of both approaches with regard to efficiency and target specificity.

Analyzing gene functions by gene knock out approaches in $A$. rhizogenes transformed root systems is also hampered by a high variability within the experimental system with independent transformation events being present in a root system after $A$. rhizogenes transformation. Hence, to facilitate investigating gene functions in non-uniformly transformed root systems, a strong expression strength of the gene knock down constructs is required. However, the widely applied $35 \mathrm{~S}$ promoter for driving knock down constructs mediates a rather weak expression strength in $M$. truncatula roots [13], with particularly weak expression in arbusculecontaining cells of mycorrhizal roots [14]. We therefore developed a vector series with three different promoters for knock down construct expression, either the $35 \mathrm{~S}$ promoter or the ubiquitin 3 promoter of Arabidopsis thaliana or the MtPt4 promoter of M. truncatula. The latter is mediating a particular strong expression in arbuscule-containing cells [15].

Arbuscules are intracellular fungal structures formed in the plant's inner cortical cell layers. The development of arbuscules requires a profound reprogramming of the root cell [16], and a wide number of genes which are specifically expressed in arbuscule containing cells have been identified $[17,18]$. However, an analysis of the precise role of these genes during arbuscule development and function is often hampered by the previously mentioned inconveniences regarding expression perturbation experiments in mycorrhizal $M$. truncatula roots.

Here we demonstrate that mtr-miR159b is effectively processed from its precursor molecule and thus represents a highly suitable backbone for the expression of amiRs in M. truncatula. Efficient target gene knock-down could be validated by an amiR against a visible marker in an heterologous system and in $M$. truncatula. Additionally, we used the MtPt4 promoter, which mediates a strong expression in mycorrhizal roots [15] for driving the expression of an amiR against a previously identified putative transcription factor (MtErf1). Knock-down of MtErf1 expression resulted in reduced expression of levels of Rhizophagus irregularis genes indicating reduced mycorrhizal colonization. Moreover, MtErf1 seemed to be required for arbuscule development, since only truncated, non-fully branched arbuscules were present in roots with amiR-silenced MtErf1 expression.

\section{Results and discussion}

miR159b represents a suitable backbone for artificial microRNA (amiR) expression in $M$. truncatula

Expression of artificial miRNAs requires a miRNA backbone sequence, of which the mature miRNA is replaced by an artificial miRNA (amiR), which binds and cleaves its target sequence(s). One prerequisite for amiR constructs is that star sequences and other small RNAs deriving from these constructs do not accumulate, and therefore could not regulate other off-targets. For correct processing of the amiR, the endogenous miRNA backbone must have a non-canonical loop-to-base processing type, with the first cleavage step, which seems to be most critical for miRNA processing occurring in the top region of the precursor independent of the miRNA sequence itself $[19,20]$. It is assumed that a clear physical separation of the first cleavage position and the miRNA sequence provides a high flexibility for manipulation of amiR sequences [19,21]. A previous study employed the $A$. thaliana loop-to-base processed miR319 as precursor for amiR expression in M. truncatula, which led to a significant downregulation of flottilin gene expression in roots [22]. However, in order to optimize expression of amiRs in $M$. truncatula, we decided to use an endogenous miRNA molecule as precursor. For this purpose, we screened our recent miRNA and degradome data of $M$. truncatula roots [23] for amiR backbone sequences and selected mtr-miR159b as a suitable precursor (Figure 1) since it showed all the necessary features mentioned above. The distribution of degradome tags across the miR159b precursor sequence confirmed the loop to base processing for this miR159 family member in M. truncatula. The miR159b primary transcript sequence was cloned in the pBluescript II SK+ vector flanked by restriction sites, which allow a directed cloning of the amiR sequences in the appropriate binary vectors for plant transformation (Additional file 1: Figure S1.)

\section{A vector system for expression perturbation experiments by $A$. rhizogenes mediated root transformation}

For reverse genetic approaches in $M$. truncatula roots, we developed a vector series (pRed), where a constitutively expressed dsRED gene allows the easy detection of transformed roots (Additional file 1: Figure S2). We have developed expression vectors (pRed-Exp) and vectors for RNAi (pRed-RNAi). Both types of vectors are available with three different promoters for expression of the gene or RNAi construct, namely the $2 \times 35 \mathrm{~S}$ promoter, the ubi3 promoter of Arabidopsis thaliana and the MtPt4 promoter of M. truncatula [15].

\section{The miR159b-mediated amiR expression mediates strong} silencing in tobacco leaves

To test the functionality of the mtr-miR159b backbone system, we first generated amiRs targeting dsRED and 


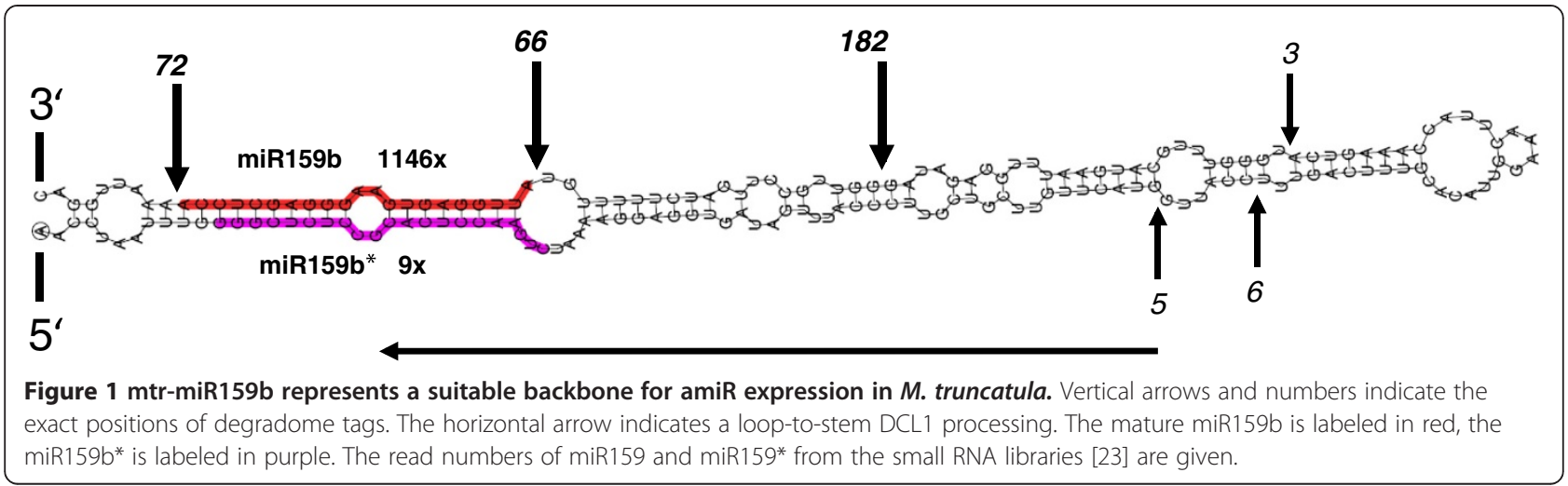

tested them in a heterologous system using dsRED fluorescence as visible marker for gene silencing. An amiR against dsRED was designed using the WMD3 web microRNA designer. Constructs were generated using an overlapping PCR strategy as recently described [12], where the mature miR159b sequence was replaced by sequences complementary to a selected region of the target gene. The novel star sequence was designed in a way that the secondary structures of the miR159b backbone were conserved. In a first attempt to test the mtr-miR159b-mediated amiR expression, we introduced $35 \mathrm{~S}_{\text {pro }}:$ amiR-dsred constructs by agroinfiltration in tobacco (Nicotiana benthamiana). Since the vector

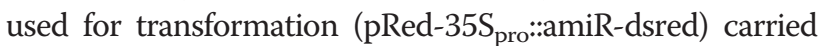
a constitutively expressed dsREDvisible marker for transformation, we expected that the amiR-dsred expression from the same vector will mediate a silencing of the dsRED. As a control we transformed the same leaves with an empty pRed-35S $S_{\text {pro }}$ vector. As expected, infiltration sites of the empty vector control showed a dsRED fluorescence, whereas infiltration of the pRed$35 \mathrm{~S}_{\text {pro }}:$ amiR-dsred construct led to only weak fluorescence (Figure 2). Analysis of the mRNA levels isolated from the infiltration sites confirmed gene silencing by the amiR-dsred. The abundance of dsRed mRNA was significantly lower in all three amiR-infiltrated leaves as compared to the vector controls (Figure 2B). Also a Western blot confirmed the significantly lower accumulation of dsRED proteins in amiR-infiltrated leaves as compared to the vector controls (Figure 2C).

\section{The miR159b backbone driven amiR constructs lead to efficient knock-down in $M$. truncatula roots}

Next, we tested the functionality of miR159b-mediated amiR expression in roots of $M$. truncatula. Stably transformed $M$. truncatula plants, which constitutively

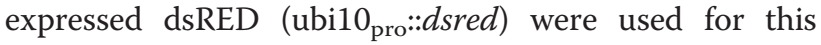
purpose. We expected a loss of dsRED expression in roots after transformation with amiR-dsred construct, which would indicate that the miR159b backbone-driven
amiR system works in M. truncatula roots. DsRed-specific amiRs were cloned behind the pORE-E4 vector [24]. As control, empty vectors were used. As expected, root transformation with the amiR-dsred construct led to the growth of non-fluorescent roots, whereas in the vector controls all of the roots kept the dsRED fluorescence (Figure 3).

\section{MtPt4 promoter driven amiR silencing of MtErf1 points to a role of this TF in arbuscule-development}

Finally we wanted to confirm that amiR-mediated gene silencing also works efficiently in arbuscule-containing cells. Since the $35 \mathrm{~S}$ promoter seems to be only weakly active in arbuscule-containing cells [14], we used the MtPt4 promoter of M. truncatula [15], to enable a strong and specific expression of the amiRs. MtPt4 encodes for a phosphate transporter, which is strongly induced in arbuscule-containing cells $[15,25]$.

As a candidate gene for the proof of concept experiment we selected MtErf1, a putative member of the AP2 (APETALA2)-EREBP (ethylene-responsive element binding protein) transcription factor family. MtErf1 (medtr7g009410) was found to be strongly and specifically expressed in arbuscule-containing cells (Figure 4A and Additional file 1: Figure S3). The gene was previously identified by $M$. truncatula transcription factor (TF) profiling [26] in order to identify TFs, which are induced in mycorrhizal roots (Reinert et al. unpublished). MtERF1 consists of 392 amino acids with two AP2 domains in the $\mathrm{N}$-terminal part of the protein. Hence, MtERF1 belongs to the subfamily of AP2 genes within the AP2-EREBP family. This is in contrast to previously described $M$. truncatula ERF transcription factors required for nod factor signal transduction $[27,28]$. It is worth mentioning, that the C-terminal part of the MtERF1 amino acid sequence seems to be only weakly conserved. However, homologous sequences were found in several plant species but not in the non-mycorrhizal plant $A$. thaliana (Additional file 1: Figure S4) supporting the assumption that MtERF1 might have specific functions 

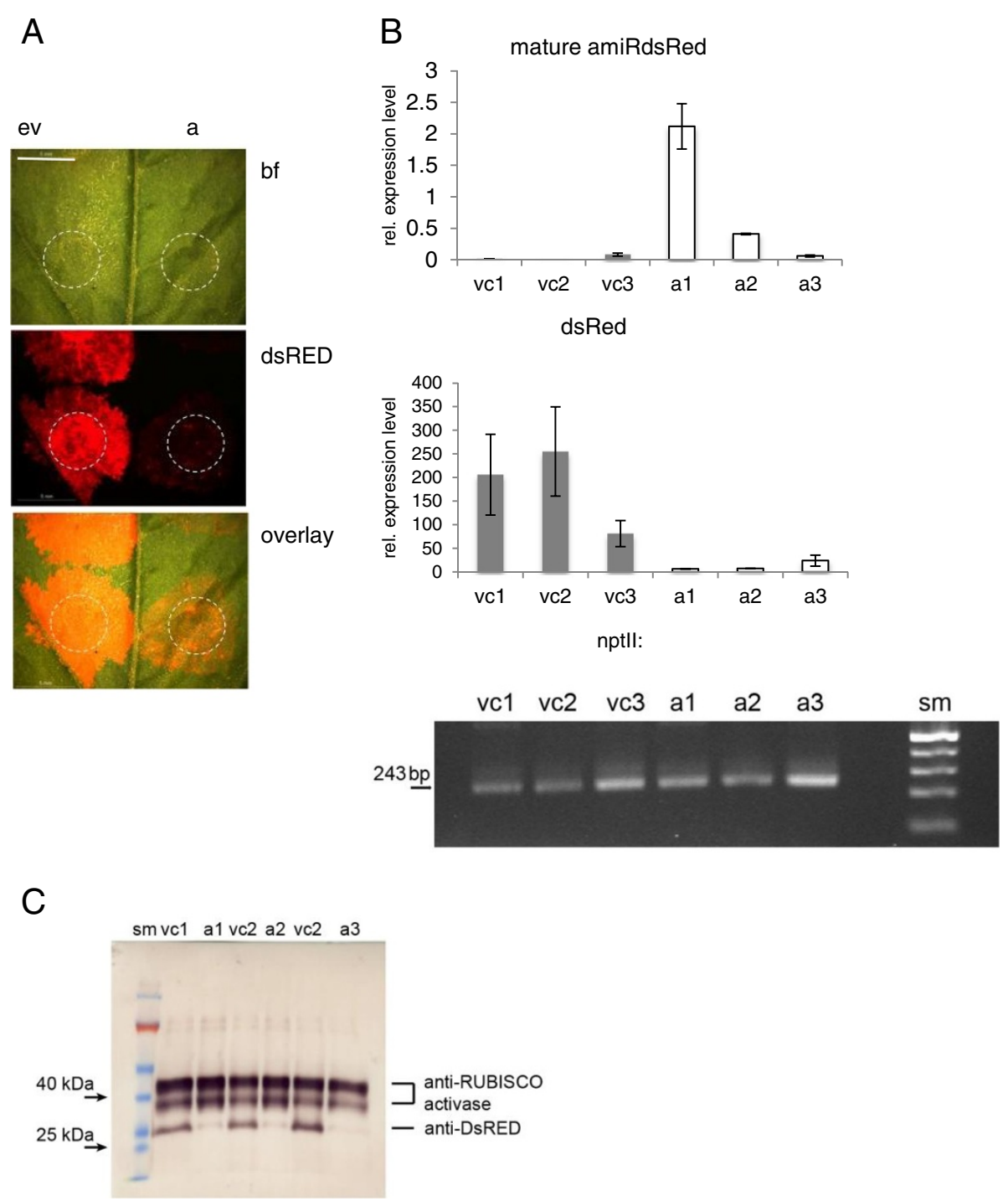

Figure 2 The $\mathrm{mt}$-miR159b-mediated amiR-silencing is efficient in tobacco leaves. A) DsRED fluorescence is strongly reduced after infiltration of the pRed-35Spro::amiR-dsred (A) construct (a) as compared to empty vectors (ev). Bright filed image (bf), DsRED fluorescence (dsRED) and an overlay of both channels are shown. Infiltration site are indicated by dotted lines. Scale bar: $5 \mathrm{~mm}$. B) Relative levels of mature amiR-dsred determined by stem loop qRT-PCR, relative transcript levels of dsred measured by qRT-PCR and transcript levels of nptll (kanamycin resistance) are shown. vc: vector controls, a: amiR samples sm: size marker, amplicon size of $243 \mathrm{bp}$ is indicated. C) Western blot demonstrating reduced expression of dsRED after amiR-dsred mediated silencing of dsRED in tobacco leaves. Shown are three replicates of amiR-silencing (a) and vector controls (vc) represented by proteins of three different infiltration spots on the same leaf, for each construct. Reduced expression of dsRED after amiR-dsred infiltration was visualized using an anti-dsRED antibody. Equal sample loading is shown by anti-RUBISCO activase.

in arbuscule development or functioning. To test this assumption, an MtErf1-specifc amiR was designed as described before and cloned behind the MtPt4 pro-

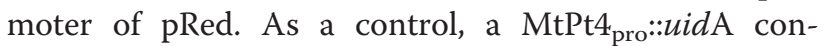
struct was used in the same vector. Roots that were transformed with the amiR construct, showed a significantly decreased expression of MtErf1 transcripts (Figure 4B). In addition, transcript levels of two diagnostic markers of a functional AM symbiosis, MtPt4 and $R$. irregularis elongation factor, were also clearly reduced in roots after amiR expression. This indicates that the MtPt4 promoter mediated an efficient amiRsilencing in arbuscule-containing cells. Moreover, reduced MtErf1 transcripts levels led to reduced expression of genes related to a functional mycorrhizal colonization and thus a lower level of mycorrhizal colonization. We next investigated the arbuscule morphology in vector control and amiR-MtErf1 roots. Arbuscule morphology was strongly impaired in amiRMtErf1 roots with only small, truncated arbuscules 


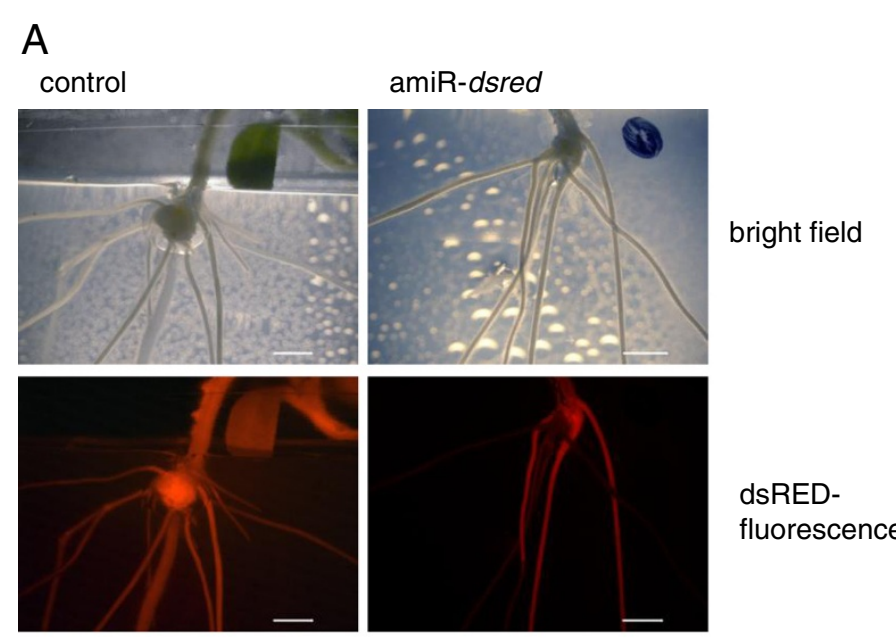

B

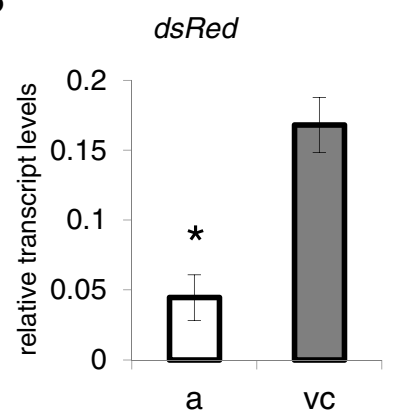

amiR-dsred

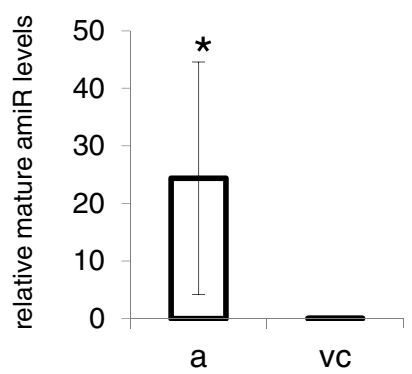

C

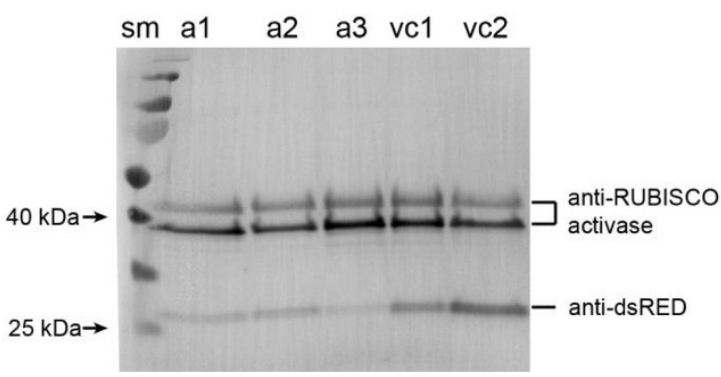
truncatula plants (35Spro::dsRED) were used for A. rhizogenes mediated root transformation. After transformation with the amiR-dsRed construct (right), parts of the roots lost dsRED fluorescence due to amiR-mediated gene silencing. Scale bar represents $5 \mathrm{~mm}$. B) Relative levels of mature amiR-dsred determined by stem loop qRT-PCR and relative transcript levels of dsred measured by qRT-PCR as determined by qRT-PCR. Values shown are mean values of 3-4 biological replicates $+/-$ standard deviation. Asterisks indicate statistical differences $(P<0.05)$. C) Western blot analysis confirmed reduced accumulation of dsRED proteins in roots after transformation with the amiR-dsRed construct (a). Shown are three replicates of amiR-silenced root samples and two vector controls (v). Reduced accumulation of dsRED protein after amiR-dsred infiltration were visualized using an anti-dsRED antibody. Equal sample loading is shown by anti-RUBISCO activase.

being present in these roots (Figure 4C). This points to a function of this putative TF in arbuscule development and indicates the MtErf1 seems to be required for a full maturation of arbuscules.

\section{Conclusions}

The endogenous amiR-mediated gene silencing system presented here provides a useful tool to investigate the function of genes involved in root endosymbioses. In addition, we showed that the MtPt4 promoter provides a strong expression of amiR constructs in mycorrhizal roots. AmiR-silencing of a putative transcription factor $M r E r f 1$ indicated a putative function of this gene during arbuscule development, since only defective arbuscules were observed in roots with reduced $M t E r f 1$ expression due to amiR-mediated gene silencing. 

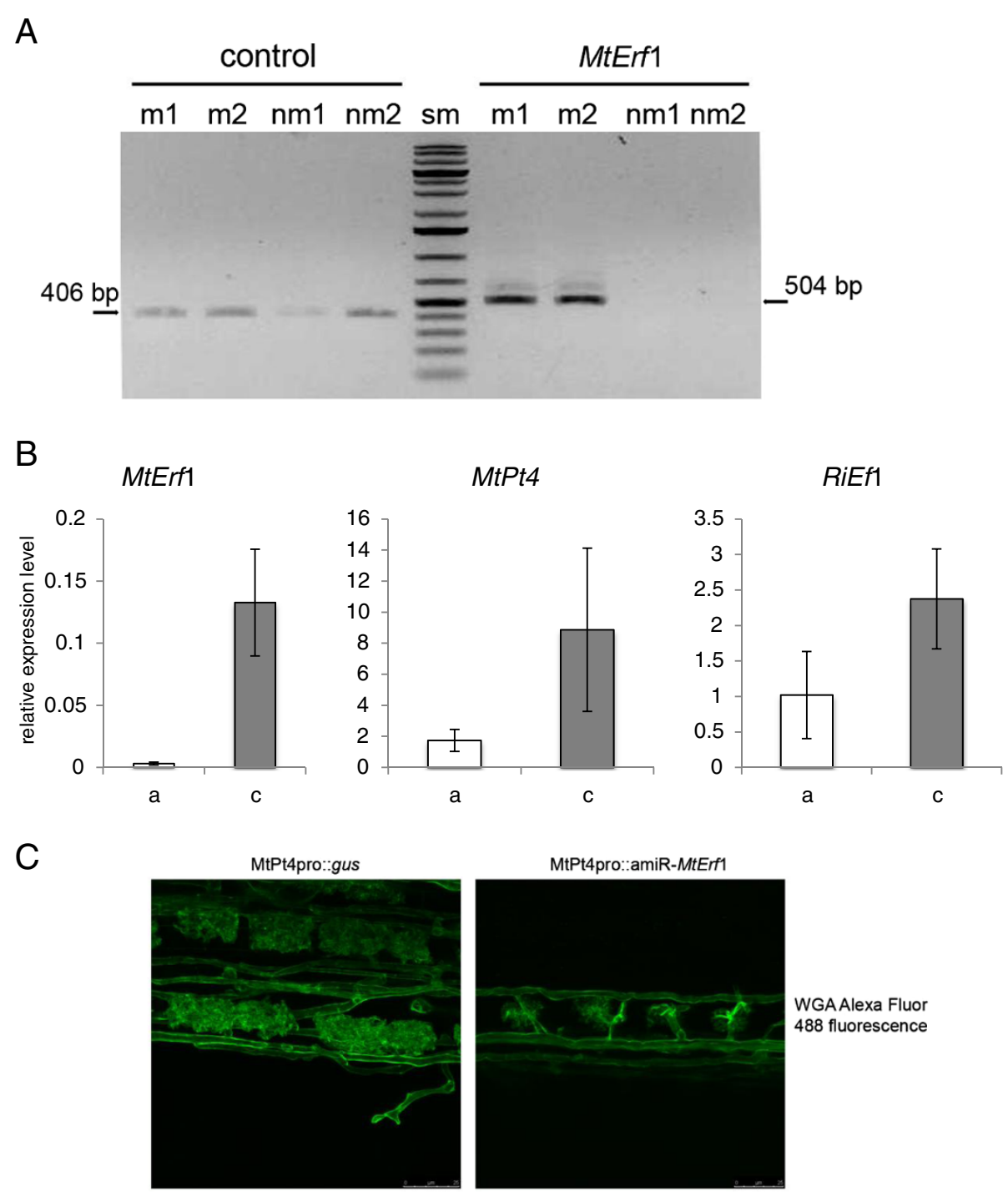

WGAAlexa Fluor 488 fluorescence
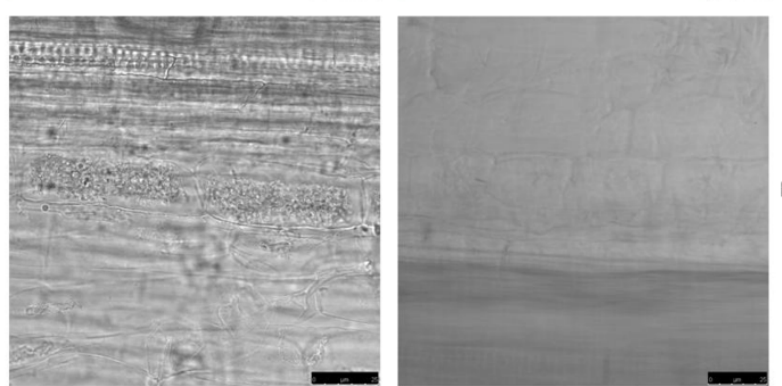

Figure 4 Artificial microRNA (amiR)-mediated silencing of DsRED expression in $\mathbf{M}$. truncatula roots. A) MtErf1 transcripts are specifically induced in mycorrhizal roots (myc) and non- detectable in non-mycorrhizal (nm) roots. MtErf1 and MtEf1 as reference (control) transcripts were amplified from CDNA of mycorrhizal and non-mycorrhizal roots of M. truncatula by RT-PCR. Amplicon sizes are indicated. Two biological replicates are shown. B) Relative levels of MtErf1, MtPt4 and R. irregularis elongation factor 1 alpha (RiEf1) [29] were determined by qRT-PCR. Values shown are means of 3-4 biological replicates $+/-$ one standard deviation. Each replicate consisted of several roots $(n<5)$ transformed with either the MtPt4pro::amiR-MtErf1 (a) construct or MtPt4pro::uidA control (c). C) amiR-mediated MtErf1 knock-down results in deformed arbuscules. Shown are representative figures of arbuscules observed in roots transformed with either the MtPt4pro:: amiR-MtErf1 construct or the MtPt4pro::uidA control. Bright field images and WGA-Alexafluor 488 fluorescence are shown. Scale bar reesents $25 \mu \mathrm{m}$. 


\section{Methods}

\section{Biological Material}

The following plants were used in this study: Nicotiana benthaminana cv. TW16, Medicago truncatula cv. Jemalong (A17) and Medicago truncatula cv. 2HA stably transformed with pKDSR(I) (see vectors and cloning procedures for vector details). Bacterial strains include Escherichia coli TOP10 (Invitrogen) or DH5 $\alpha$ for cloning purposes, Agrobacterium rhizogenes strain ARqua1 [30] for Medicago root transformations and Agrobacterium tumefaciens strain GV2260 [31] for the leaf infiltration assay. The fungus Rhizophagus irregularis (strain BB-E, provided by Agrauxine, France) was propagated and used for mycorrhizal colonization studies as described in [23].

\section{Medicago truncatula growth, transformation and inoculation}

Medicago truncatula seeds were scarified using concentrated $\mathrm{H}_{2} \mathrm{SO}_{4}$ and subsequently sterilized with $\mathrm{HClO}$. The seeds were laid on a water agar plate and kept at $4^{\circ} \mathrm{C}$ in the dark overnight to synchronize the germination. Afterwards, the seeds were transferred to room temperature but remained in the dark for two days. $M$. truncatula root transformation was carried out with a modified protocol according to [32]. Seedlings were subsequently transferred to a fresh water agar plate, kept in the dark and incubated at room temperature for a further two days. Finally the seedlings were transferred into vertical square plates (amiRdsRED) or jars (amiRMtErf1) containing Fahraeus medium plus $25 \mu \mathrm{g} / \mathrm{ml}$ kanamycin. The plates and jars were kept in a phytotron for three weeks to allow the growth of transformed roots. The phytotron conditions were: $200 \mu \mathrm{E} \cdot \mathrm{m}^{-2} \cdot \mathrm{s}^{-1}, 16 \mathrm{~h} / 8 \mathrm{~h}$ daynight cycle, $22^{\circ} \mathrm{C}$ and $65 \%$ humidity. After three weeks the plants expressing the amiR- and control construct indicated by a DsRed fluorescence, were potted in a soil mix of quartz sand (0.6-1.2 mm)/expanded clay/vermiculite/inoculum mix $(7: 1: 1[\mathrm{v} / \mathrm{v}])$. All potted plants were fertilized twice a week with $20 \mu \mathrm{M} \mathrm{P}_{\mathrm{i}}$ Hoagland's solution [33].

M. truncatula Jemalong, genotype 2HA was used for plant transformation with the vector $\operatorname{pKDSR}(\mathrm{I})$ (see vectors) [34].

\section{Leaf infiltration assays}

Nicotiana benthamiana plants were grown for 4 weeks in a phytotron and used for the infiltration prior to the flowering phase. The experiment was repeated on two individual plants and six infiltrations each. Prior to infiltration, the plants were watered for $3 \mathrm{~h}$ until the soil was water saturated. The leaves were infiltrated either with Agrobacterium tumefaciens (GV2260) containing pRed$35 \mathrm{~S}_{\text {pro: }}:$ amiR-dsred or empty pRed-Expr. The bacteria were harvested, washed with AS-media (10 mM MES,
$10 \mathrm{mM} \mathrm{MgCl}_{2}, \mathrm{pH}$ 5.6), resuspended to an $\mathrm{OD}_{600}$ of 0.8 with AS-media containing $150 \mu \mathrm{M}$ acetosyringone and incubated for $3 \mathrm{~h}$ at room temperature on a shaker (50 rpm). Using a syringe, $500 \mu \mathrm{l}$ of the bacterial suspension was infiltrated into the abaxial side of each leaf. Infiltration boarders were marked with a permanent marker. The plants were placed into a phytotron and analyzed after two and three days. After analysis, the marked leaf areas were excised, frozen in liquid nitrogen and stored at $-80^{\circ} \mathrm{C}$ for further protein and RNA extraction.

\section{Staining and determination of fungal structures}

Fungal structures were visualized with wheat germ agglutinin (WGA) conjugated with Alexa Fluor 488 (Invitrogen). In short, the approx. $1 \mathrm{~cm}$ long root $\mathrm{sec}$ tions were submerged for $5 \mathrm{~min}$ in $90^{\circ} \mathrm{C}$ hot $10 \% \mathrm{KOH}$ [w/v] and washed five times with phosphate buffered saline (PBS) buffer (pH 7.4). The roots were then incubated overnight in PBS buffer (pH 7.4) containing 0.01\% WGA Alexa Fluor 488 [w/v].

\section{Fluorescence imaging}

The DsRed fluorescence of tobacco leaves and the roots of chimeric $M$. truncatula plants were monitored using a stereo-fluorescence microscope Leica M165 FC with a DsRed filter system (Leica 10447227). The exposure time for fluorescence was $500 \mathrm{~ms}$ and the gain setting was $2.0 \times$. Overlay images were produced using Leica LAS-AF Version 2.8.1.

Confocal Images of WGA-Alexa Fluor stained arbuscules were collected on a Leica TCS-SP5 confocal microscope (Leica Microsystems, Exton, PA USA) using a $63 \times$ water immersion objective NA 1.2, zoom 1.6. Alexa Fluor was excited at $488 \mathrm{~nm}$ and emitted light was collected from 505 to $582 \mathrm{~nm}$. Optical sections were acquired at $0.3-0.5 \mu \mathrm{m}$ intervals. Images were processed using Image J software (Wayne Rasband, National Institutes of Health, USA; http//imagej.nih.gov/ij).

\section{Mapping of small RNA and degradome reads to miRNA precursors and design of artificial miRNAs}

All $M$. truncatula precursor sequences belonging to the families of miR159 and miR319, were gathered and analyzed for small RNA and degradome read location as well as abundance. Small RNA and degradome reads were previously mapped to the Medicago 3.0 genome [23]. The information of the precise location of the reads and their abundance was manually annotated to the appropriate precursor. The 21 nt long artificial miRNA sequences against $d s R E D$ and $M t E r f 1$ were designed using the web miRNA designer WMD3 (http:// wmd3.weigelworld.org/cgi-bin/webapp.cgi) following the instructions given on the website. Only green 
flagged sequences were chosen and checked against their target sequence and the Medicago genome v3.5 using psRNAtarget (http://plantgrn.noble.org/psRNATarget/). The sequence showing no or the least possible offtargets was chosen for further construction of the artificial miRNA.

\section{Vectors and cloning procedure}

The vector $\mathrm{pKDSR}(\mathrm{I})$ was created by converting the unique AscI of pRedroot [35] to a PacI site, in a way that the gene with its promoter and terminator was flanked by two PacI sites. Likewise, a KpnI site of pK7GWIWG2 (I) [36] was converted into a PacI site. The PacI flanked cassette was excised from pRedroot and ligated into the novel PacI site of pK7GWIWG2(I). The resulting vector was named pKDSR(I).

For the construction of the pRed-amiR vectors, the final amplification product of the overlapping PCR was first cloned into pCR2.1 (Invitrogen). The PCR product included parts of the pBluescript II SK (+) multiple cloning site. The amiR precursor molecule was removed from the pCR2.1 vector by using the SpeI and MluI restriction sites and ligated into the appropriate sites of pRed vectors. Final pRed-amiR constructs were sequenced and used for root transformation (pRed-35Spro::amiR-dsred, pRed-MtPt4pro:: amiR-MtErf1) as described recently [32]. Additionally, for tobacco leaf infiltration assays, the amiR-dsred precursor molecule was inserted via NotI and KpnI sites into pORE-E4 [24].

\section{Overlapping PCR using the miR159b backbone to design an artificial miRNA}

The miR159b backbone was synthesized by gene synthesis (MWG) and an additional MluI site was added to the 3 ' end of the miR precursor. This molecule was cloned into the SpeI and PstI restriction site of pBluescript II SK (+). The resulting $\mathrm{pB} 159 \mathrm{~b}$ vector represents the template for the overlapping PCR to generate amiR precursor molecules. For this purpose four different primers are designed according to [37].

Primer I: GTX ${ }_{1} \ldots \mathrm{X}_{21}$ AAATTGGACACGCGTct $\left(\mathrm{X}_{1}-\mathrm{X}_{21}\right.$ are the designed amiR sequence).

Primer II: TTY $_{1} \ldots Y_{21}$ ACAAAAAGATCAAGGC $\left(\mathrm{Y}_{1}-\mathrm{Y}_{21}\right.$ are the amiR sequence in reverse complement orientation).

Primer III: $\mathrm{TTZ}_{1} \ldots \mathrm{Z}_{21}$ TCTAAAAGGAGGTGATAG $\left(Z_{1}-Z_{21}\right.$ are the amiR sequence in reverse complement orientation, with the exception that $Z_{11}$ and $Z_{12}$ have to be modified to not pair (also no G:U non-Watson-Crick pairing) to position $X_{10}$ and $X_{11}$, respectively. Also $Z_{21}$ has to be changed to not pair to $X_{1}$.

Primer IV: GAN $\mathrm{G}_{1} \ldots \mathrm{N}_{21}$ AATTAGGTTactagt $\left(\mathrm{N}_{1}-\mathrm{N}_{21}\right.$ are reverse complement of $\left.Z_{1}-Z_{21}\right)$. Primer sequences used to create amiRDsRED and amiRMtErf1 are given in the Additional file 2: Table S1.

Three independent PCRs were performed with pB159b as template and the following three primer combinations (1) primer A + primer I, (2) primer II + primer III, (3) primer $\mathrm{B}+$ primer IV. The PCR products were loaded into a single well of an $2 \%$ agarose gel followed by gel purification. The resulting mixture of products was used as a template for a final PCR using primer A + primer B. The single PCR product was subcloned into pCR2.1 (Invitrogen) and sequenced to check for a correct amiR precursor sequence and a stem-loop folding identical to mtr-miR159b.

\section{RNA extraction, RT-PCR and quantitative RT-PCR}

Total RNA was extracted from liquid nitrogen frozen and ground tissue using the miRVana miRNA extraction Kit (Ambion) and Plant Isolation Aid step (Ambion) according to the manufacturer's instructions. All PCRs were carried out as described earlier in $[23,38]$.

\section{Protein extraction and Western blotting}

Frozen plant tissue was ground and proteins were extracted with rigorous vortexing in $4 \mathrm{ml}$ of homogenization buffer per gram fresh weight. The homogenization buffer consisted of 100 mM HEPES pH 7.5, 10\% glycerol, 5 mM DTT, cOmplete ULTRA tablet - EDTA free (Roche) proteinase inhibitor cocktail. The extract was centrifuged at $14000 \mathrm{~g}$ for $15 \mathrm{~min}\left(4^{\circ} \mathrm{C}\right)$ and the supernatant was collected and aliquots were frozen at $-20^{\circ} \mathrm{C}$. The protein concentration was determined using QuickStart Bradford Protein Assay (Biorad) with the provided $\gamma$-globulin standard following the manufacturer's instructions using the microtiter plate protocol.

For the western blot analysis an equal amount of $15 \mu \mathrm{g}$ of protein from extracts of tobacco plants and $10 \mu \mathrm{g}$ of proteins from Medicago root extracts were resolved on separately on $1 \mathrm{~mm}$ 12\% SDS-PAGE mini-gels, respectively, and subsequently transferred onto Immobilon-P PVDF membranes (Millipore) by semidry blotting according to Immobilon-P transfer membrane user guide. Detection of DsRed was carried out with primary antibodys using $0.4 \mu \mathrm{g} / \mathrm{ml}(1: 1250)$ rabbit anti-RFP tag antibody (GenScript) mixed with rabbit anti-RubisCO activase (Agrisera) in a 1:10000 dilution, the latter serving to detect RubisCO activase as a loading control. The secondary goat anti-rabbit antibody conjugated with alkaline phosphatase (Abcam) was used as a 1:10000 dilution. The protein bands were visualized using NBT/BCIP (Roche). The specific proteins were identified by comparison to a pre-stained protein size marker (Thermo scientific). 


\section{Statistics}

To test for difference between plant genotype and treatment, data were analyzed by Student's $t$-test for pairwise comparisons using the Sigmaplot software package (Systat, Germany).

\section{Additional files}

Additional file 1: Figure S1. Sequence of the mtr-miR159b backbone for amiRNA expression in pBluescriptll SK+ vector. AmiRNA constructs are generated from this template using an overlapping PCR strategy (Schwab et al., 2006; Ossowski et al., 2008; Schwab et al., 2010). An additional Mlul restriction site was introduced for subcloning into the pRed vectors.

Figure S2. Schematic representation of the T-DNA of the pRed vector series. Vectors are available as expression vectors (amiR expression) or RNAi vectors. An ubiquitin10 (ubi10) promoter-driven dsred gene allows the identification of transformed roots via the dsred fluorescence. The MtPt4 promoter mediates a strong expression in arbuscule-containing cells (pRed-PT4). Alternatively, the ubiquitin10 (pRed-ubi3 ) or $2 \times 35 \mathrm{~S}$ (pRed-35S) promoter mediate a constitutive expression the corresponding constructs. TL/R: left/right border of T-DNA, pro: promoter, nptll: neomycin phosphotransferase (kanamycin resistance) ter: terminator, OCS: Octopin synthase. Figure S3. Activity of the MtErf1 promoter in arbuscule-containing cells of mycorrhizal roots of $M$. truncatula. 1145 bp MtErf1 promoter region were fused to cyan fluorescent protein (CFP). and transferred by Agrobacterium rhizogenes transformation into $M$. truncatula roots. Roots were colonized with $R$. irregularis. Three weeks after inoculation, roots were harvested and CFP fluorescence was observed in cross section. Fungal structures were stained with WGA- Alexafluor 594. Arrowheads indicate arbuscules, iH: intercellular hyphae, P: pericycle, scale bars indicate $25 \mu \mathrm{m}$. Figure S4. Alignments of MtERF1 wit homologs from Glycine max Gm_1 (XP_003533548.1), Gm_2 (XP_003551723.1), Gm_3 (XP_003530686.1), Gm_4 (XP_003553203.1), Populus trichocarpa Pt_1 (XP_002323836.1 ), Pt_2 (XP_002315794.1), Ricinus communis Rc_1 (XP_002517474.1), Vitis vinifera Vv_1(CAN79925.1), Vv_2 (XP_003633849.1), Vv_3 (XP_002270149.1), Fragaria vesca Fv_1(XP_004304943.1), Fv_2 (XP_004298447.1), Prunus persica Pp_1 (EMJ17977.1),Pp_2 (EMJ18018.1),Pp_3 (EMJ27523.1), Cucumis sativus Cs_1 (XP_004147491.1). The two AP2 domains are underlined in red and identical positions are highlighted in grey.

Additional file 2: Table S1. Primer and amiR sequences used in this study.

\section{Competing interest}

The authors declare no competing interests.

\section{Authors' contributions}

EAD and JT carried out the molecular analyses, participated in the design of the study, performed the statistical analysis and drafted the manuscript. AR carried out the promoter reporter assays. NG carried out the stable plant transformation. FK conceived of the study, and participated in its design and coordination and helped to draft the manuscript. All authors read and approved the final manuscript.

\section{Acknowledgements}

The Max Planck Society supported this work. We thank Igor Kryvoruchko for constructing the pKDsRed vector. We thank Ursula Krause for providing the anti-RubisCO activase antibody and Derek Nedveck for critical reading of the manuscript.

\section{Author details}

${ }^{1}$ Max Planck Institute of Molecular Plant Physiology, Am Muehlenberg 1 14476, Potsdam (OT) Golm, Germany. ${ }^{2}$ Swiss Federal Institute of Technology Zurich, Department of Biology, Zurich, Switzerland.

Received: 11 February 2013 Accepted: 10 May 2013

Published: 16 May 2013

\section{References}

1. Waterhouse PM, Graham MW, Wang MB: Virus resistance and gene silencing in plants can be induced by simultaneous expression of sense and antisense RNA. Proc Natl Acad Sci U S A 1998, 95(23):13959-13964.

2. Smith NA, Singh SP, Wang MB, Stoutjesdijk PA, Green AG, Waterhouse PM: Total silencing by intron-spliced hairpin RNAs. Nature 2000, 407(6802):319-320.

3. Small I: RNAi for revealing and engineering plant gene functions. Curr Opin Biotechnol 2007, 18(2):148-153.

4. Baulcombe D: RNA silencing in plants. Nature 2004, 431(7006):356-363

5. Baulcombe D: RNA silencing. Trends Biochem Sci 2005, 30(6):290-293.

6. Kjemtrup S, Sampson KS, Peele CG, Nguyen LV, Conkling MA, Thompson WF, Robertson D: Gene silencing from plant DNA carried by a Geminivirus. The Plant journal: for cell and molecular biology 1998, 14(1):91-100.

7. Wesley SV, Helliwell CA, Smith NA, Wang MB, Rouse DT, Liu Q, Gooding PS, Singh SP, Abbott D, Stoutjesdijk PA, et al: Construct design for efficient, effective and high-throughput gene silencing in plants. The Plant journal: for cell and molecular biology 2001, 27(6):581-590.

8. Melito S, Heuberger AL, Cook D, Diers BW, MacGuidwin AE, Bent AF: A nematode demographics assay in transgenic roots reveals no significant impacts of the Rhg1 locus LRR-Kinase on soybean cyst nematode resistance. BMC Plant Biol 2010, 10:104.

9. Xu P, Zhang Y, Kang L, Roossinck MJ, Mysore KS: Computational estimation and experimental verification of off-target silencing during posttranscriptional gene silencing in plants. Plant Physiol 2006, 142(2):429-440.

10. Brodersen $P$, Voinnet $O$ : The diversity of RNA silencing pathways in plants. Trends Genet 2006, 22(5):268-280.

11. Ossowski S, Schwab R, Weigel D: Gene silencing in plants using artificial microRNAs and other small RNAs. The Plant journal: for cell and molecular biology 2008, 53(4):674-690.

12. Schwab R, Ossowski S, Warthmann N, Weigel D: Directed gene silencing with artificial microRNAs. Methods Mol Biol 2010, 592:71-88.

13. Auriac MC, Timmers AC: Nodulation studies in the model legume Medicago truncatula: advantages of using the constitutive EF1alpha promoter and limitations in detecting fluorescent reporter proteins in nodule tissues. Molecular plant-microbe interactions: MPMI 2007, 20(9):1040-1047.

14. Pumplin N, Zhang X, Noar RD, Harrison MJ: Polar localization of a symbiosis-specific phosphate transporter is mediated by a transient reorientation of secretion. Proc Natl Acad Sci U S A 2012, 109(11):E665-E672.

15. Harrison MJ, Dewbre GR, Liu J: A phosphate transporter from Medicago truncatula involved in the acquisition of phosphate released by arbuscular mycorrhizal fungi. Plant Cell 2002, 14(10):2413-2429.

16. Harrison MJ: Cellular programs for arbuscular mycorrhizal symbiosis. Curr Opin Plant Biol 2012, 15(6):691-698.

17. Gaude N, Bortfeld S, Duensing N, Lohse M, Krajinski F: Arbusculecontaining and non-colonized cortical cells of mycorrhizal roots undergo extensive and specific reprogramming during arbuscular mycorrhizal development. The Plant journal: for cell and molecular biology 2012, 69(3):510-528.

18. Hogekamp C, Arndt D, Pereira PA, Becker JD, Hohnjec N, Küster H: Laser microdissection unravels cell-type-specific transcription in arbuscular mycorrhizal roots, including CAAT-box transcription factor gene expression correlating with fungal contact and spread. Plant Physiol 2011, 157(4):2023-2043.

19. Bologna NG, Mateos $J$, Bresso EG, Palatnik JF: A loop-to-base processing mechanism underlies the biogenesis of plant microRNAs miR319 and miR159. EMBO J 2009, 28(23):3646-3656.

20. Schwab R, Voinnet O: miRNA processing turned upside down. EMBO J 2009, 28(23):3633-3634.

21. Bologna NG, Schapire AL, Palatnik JF: Processing of plant microRNA precursors. Brief Funct Genomics 2013, 12(1):37-45.

22. Haney $\mathrm{CH}$, Long SR: Plant flotillins are required for infection by nitrogen-fixing bacteria. Proc Natl Acad Sci U S A 2010, 107(1):478-483.

23. Devers EA, Branscheid A, May P, Krajinski F: Stars and symbiosis: microRNA- and microRNA*-mediated transcript cleavage involved in arbuscular mycorrhizal symbiosis. Plant Physiol 2011, 156(4):1990-2010. 
24. Coutu C, Brandle J, Brown D, Brown K, Miki B, Simmonds J, Hegedus DD: PORE: a modular binary vector series suited for both monocot and dicot plant transformation. Transgenic Res 2007, 16(6):771-781.

25. Javot H, Penmetsa RV, Terzaghi N, Cook DR, Harrison MJ: A Medicago truncatula phosphate transporter indispensable for the arbuscular mycorrhizal symbiosis. Proc Natl Acad Sci U S A 2007, 104(5):1720-1725.

26. Kakar K, Wandrey M, Czechowski T, Gaertner T, Scheible W-R, Stitt M, Torres-Jerez I, Xiao Y, Redman J, Wu H, et al: A community resource for high-throughput quantitative RT-PCR analysis of transcription factor gene expression in Medicago truncatula. Plant Methods 2008, 4(1):18.

27. Middleton PH, Jakab J, Penmetsa RV, Starker CG, Doll J, Kalo P, Prabhu R, Marsh JF, Mitra RM, Kereszt A, et al: An ERF transcription factor in Medicago truncatula that is essential for Nod factor signal transduction. Plant Cell 2007, 19(4):1221-1234.

28. Cerri MR, Frances L, Laloum T, Auriac MC, Niebel A, Oldroyd GE, Barker DG, Fournier J, de Carvalho-Niebel F: Medicago truncatula ERN transcription factors: regulatory interplay with NSP1/NSP2 GRAS factors and expression dynamics throughout rhizobial infection. Plant Physiol 2012, 160(4):2155-2172

29. Helber N, Wippel K, Sauer N, Schaarschmidt S, Hause B, Requena N: A versatile monosaccharide transporter that operates in the arbuscular mycorrhizal fungus Glomus sp is crucial for the symbiotic relationship with plants. Plant Cell 2011, 23(10):3812-3823.

30. Quandt HJ, Pühler AIB: Transgenic root nodules of Vicia hirsuta: A fast and efficient system for the study of gene expression in indeterminatetype nodules. Mol Plant Microbe Interact 1993, 6:699-706.

31. Deblaere R, Bytebier B, Degreve H, Deboeck F, Schell J, Vanmontagu M, Leemans J: Efficient Octopine Ti Plasmid-Derived Vectors for Agrobacterium-Mediated Gene-Transfer to Plants. Nucleic Acids Res 1985 13(13):4777-4788.

32. Boisson-Dernier A, Chabaud M, Garcia F, Becard G, Rosenberg C, Barker DG Agrobacterium rhizogenes-transformed roots of Medicago truncatula for the study of nitrogen-fixing and endomycorrhizal symbiotic associations. Mol Plant Microbe Interact 2001, 14(6):695-700.

33. Hoagland DR, Arnon DI: The water-culture method of growing plants without soil. Calif Agr Expt Sta Circ 1950, 374:1-32.

34. Chabaud M, Larsonneau C, Marmouget C, Huguet T: Transformation of barrel medic (Medicago Truncatula Gaertn) by Agrobacterium tumefaciens and regeneration via somatic embryogenesis of transgenic plants with the Mtenod12 nodulin promoter fused to the Gus reporter gene. Plant Cell Rep 1996, 15(5):305-310.

35. Limpens E, Ramos J, Franken C, Raz V, Compaan B, Franssen H, Bisseling T, Geurts R: RNA interference in Agrobacterium rhizogenes-transformed roots of Arabidopsis and Medicago truncatula. J Exp Bot 2004 55(399):983-992.

36. Karimi M, Inze D, Depicker A: GATEWAY vectors for Agrobacteriummediated plant transformation. Trends Plant Sci 2002, 7(5):193-195.

37. Schwab R, Ossowski S, Riester M, Warthmann N, Weigel D: Highly specific gene silencing by artificial microRNAs in Arabidopsis. Plant Cell 2006, 18(5):1121-1133.

38. Branscheid A, Sieh D, Pant BD, May P, Devers EA, Elkrog A, Schauser L, Scheible WR, Krajinski F: Expression pattern suggests a role of MiR399 in the regulation of the cellular response to local Pi increase during arbuscular mycorrhizal symbiosis. Molecular plant-microbe interactions: MPMI 2010, 23(7):915-926.

doi:10.1186/1471-2229-13-82

Cite this article as: Devers et al:: An endogenous artificial microRNA system for unraveling the function of root endosymbioses related genes in Medicago truncatula. BMC Plant Biology 2013 13:82.

\section{Submit your next manuscript to BioMed Central and take full advantage of:}

- Convenient online submission

- Thorough peer review

- No space constraints or color figure charges

- Immediate publication on acceptance

- Inclusion in PubMed, CAS, Scopus and Google Scholar

- Research which is freely available for redistribution

Submit your manuscript at www.biomedcentral.com/submit
C Biomed Central 